\title{
Gamma Spectrometry of Inhomogeneous Samples Using Peak-Ratio Method
}

\author{
Thomas M. Semkow ${ }^{1,2}$, Liang T. Chu ${ }^{1,2}$, Adam G. Burn ${ }^{1,2}$ \\ ${ }^{1}$ Wadsworth Center, New York State Department of Health, Empire State Plaza, Albany, NY, USA \\ ${ }^{2}$ Department of Environmental Health Sciences, School of Public Health, University at Albany, State University of New York, \\ Rensselaer, NY, USA \\ Email: thomas.semkow@health.ny.gov
}

How to cite this paper: Semkow, T.M., Chu, L.T. and Burn, A.G. (2021) Gamma Spectrometry of Inhomogeneous Samples Using Peak-Ratio Method. Journal of Applied Mathematics and Physics, 9, 2641-2659. https://doi.org/10.4236/jamp.2021.911170

Received: September 22, 2021

Accepted: November 2, 2021

Published: November 5, 2021

Copyright (c) 2021 by author(s) and Scientific Research Publishing Inc. This work is licensed under the Creative Commons Attribution International License (CC BY 4.0).

http://creativecommons.org/licenses/by/4.0/

\section{(c) (i) Open Access}

\begin{abstract}
In gamma spectrometry of voluminous samples, inhomogeneous distribution of radioactivity caused by the presence of hot particles can create significant Bias in the results of activity determinations. We developed a novel method to reduce this Bias using the gamma-peak ratio. We show that the peak area ratio of two gamma peaks of different energies, emitted by the same radionuclide, is a sensitive measure of emitting source location and thus the inhomogeneity. A new calibration formula was then derived for true gamma efficiency $p_{i}$ as a function of efficiency ratio $p_{i} / p_{j}$ of two peaks. This approach was verified by Monte Carlo simulations for a sample of $1-\mathrm{L}$ volume containing from 1 up to 2048 of hot particles randomly distributed in a soil matrix. A ${ }^{152} \mathrm{Eu}$ radionuclide was selected for calculations and we used various combinations of two gamma spectral peaks selected from three gamma energies of $121.8,344.3$, and $1408.0 \mathrm{keV}$. This new method is shown to reduce the Bias range and Bias standard deviation by several times when compared with the traditional homogeneous calibration applied to measuring hot particles. The method is independent of the number, location, and distribution of hot particles in the samples, and can be applied to a mixture of radionuclides. It complements our previous calibration model based on the peak-to-total ratio.
\end{abstract}

\section{Keywords}

Monte Carlo Simulation, Gamma Attenuation, Hot Particle, Effective Peak Efficiency, Signal Detection Theory

\section{Introduction}

In gamma spectrometry of voluminous samples, Germanium (Ge) detector calibration is normally accomplished assuming the homogeneous distribution of ra- 
dioactivity using either the physical traceable standards or computational methods. In many types of samples, however, the distribution of radioactivity may be inhomogeneous owing to the presence of hot particles, potentially leading to substantial Bias in activity determination. In the previous investigation [1], we provided a review of the sources, distribution, and fate of radioactively hot particles originating mostly from nuclear reactor accidents. Hot particles are often refractory and physical homogenization of a sample may result only in redistribution of the particles.

To address measurements of hot particles by gamma spectrometry, statistical methods have been developed based on either splitting of a large sample and measurements of several sub-samples [2] [3], or repetitive mixing and measuring of the same sample (from 25 to 100 times) [4] [5] [6]. While these methods can provide a relatively accurate average of the measured activity, they are not practical for many samples, and the dispersion evaluated by means of a standard deviation can be large and increase as the number of hot particles decreases. With a large standard deviation, significant Bias could result from a single measurement of the sample.

Thus, in this paper, we would like to answer the question: can a single laboratory measurement of an inhomogeneous sample, with proper calibration, reduce the dispersion and Bias of the results? We propose a novel calibration of the form

$$
p=f(q),
$$

where $p$ is the true gamma peak efficiency and $q$ is a parameter sensitive to inhomogeneity, which can be both calculated and measured, having a functional form of $f$. In the previous investigation [1], we used the peak-to-total ratio, $q=$ $p / t$, where $t$ is the total gamma efficiency. $p / t$ can be easily measured as the ratio of gamma peak area in counts to the total counts in the gamma spectrum.

In this paper, we propose $q=p_{i} / p_{j}$ as the ratio of two effective peak efficiencies for two gamma rays of different energies, $i$ and $j$, emitted by the same radionuclide. This ratio is experimentally realized as the ratio of corresponding peak areas or counting rates in the gamma spectrum. Since two gamma rays of different energies are attenuated differently in the sample, the ratio is expected to depend on the location of the emitting radionuclide in the sample, and thus the inhomogeneity. Gamma-peak ratios were used as one of the several techniques in the context of in-situ gamma spectrometry [7]-[12]. The goal of in-situ gamma spectrometry is to determine radioactive contamination in the field, with certain assumptions about contamination profiles such as uniform, plane, multi-layered, or exponential.

There are many multi-peak radionuclides to which peak-ratio calibration could apply. Some of the radionuclides of interest are included in Table 1, such as fission products, activation products, and natural radionuclides. Their principal gamma peak energies and intensities are listed. For the purpose of this work, we chose ${ }^{152} \mathrm{Eu}$ and selected three gamma peaks for the investigation: at 121.8, 
Table 1. Nuclear data of selected multi-peak radionuclides for the peak-ratio method [20].

\begin{tabular}{|c|c|c|c|c|}
\hline Radionuclide & Half-life & Gamma energy (keV) & Gamma intensity (\%) & Progeny \\
\hline \multirow{2}{*}{$\mathrm{I}-131$} & \multirow{2}{*}{$8.025 \mathrm{~d}$} & 364.5 & 81.50 & \\
\hline & & 637.0 & 7.16 & \\
\hline \multirow{2}{*}{ Ba-133 } & \multirow{2}{*}{$10.55 \mathrm{y}$} & 81.0 & 32.90 & \\
\hline & & 356.0 & 62.05 & \\
\hline \multirow{3}{*}{ Cs-134 } & \multirow{3}{*}{$2.065 \mathrm{y}$} & 569.3 & 15.37 & \\
\hline & & 604.7 & 97.62 & \\
\hline & & 795.9 & 85.46 & \\
\hline \multirow{2}{*}{ Cs-137(a) } & \multirow{2}{*}{$30.08 \mathrm{y}$} & 32.1 & 5.63 & \multirow{2}{*}{ Ba-137 } \\
\hline & & 661.7 & 85.10 & \\
\hline \multirow{3}{*}{ Eu-152 } & \multirow{3}{*}{$13.52 \mathrm{y}$} & 121.8 & 28.41 & \\
\hline & & 344.3 & 26.59 & \\
\hline & & 1408.0 & 20.85 & \\
\hline \multirow{4}{*}{ Ir-192 } & \multirow{4}{*}{$73.83 \mathrm{~d}$} & 296.0 & 28.71 & \\
\hline & & 316.5 & 82.86 & \\
\hline & & 468.1 & 47.84 & \\
\hline & & 604.4 & 8.22 & \\
\hline \multirow{5}{*}{$\mathrm{Ra}-226(\mathrm{a})$} & \multirow{5}{*}{$1600 \mathrm{y}$} & 295.2 & 18.42 & \multirow{2}{*}{$\mathrm{Pb}-214$} \\
\hline & & 351.9 & 35.60 & \\
\hline & & 609.3 & 45.49 & \multirow{3}{*}{ Bi-214 } \\
\hline & & 1120.3 & 14.92 & \\
\hline & & 1764.5 & 15.30 & \\
\hline \multirow{5}{*}{$\mathrm{Ra}-228(\mathrm{a})$} & \multirow{5}{*}{$5.75 y$} & 338.3 & 11.27 & \multirow{2}{*}{ Ac- 228} \\
\hline & & 911.2 & 25.80 & \\
\hline & & 238.6 & 43.60 & $\mathrm{~Pb}-212$ \\
\hline & & 583.2 & 30.55 & \multirow{2}{*}{ Tl-208 } \\
\hline & & 2614.5 & 35.85 & \\
\hline
\end{tabular}

a: In equilibrium with the progeny.

344.3, and $1408.0 \mathrm{keV}$ to study three combinations of gamma rays using Equation (1).

The approach taken in this investigation is by Monte Carlo (MC) simulation, described in detail in Section 2. We develop two calibration models: 1-particle model and an $n$-particle model. For the 1-particle model, Equation (1) is derived using gamma attenuation [13], and its dependence on calibration is described in Section 3. The n-particle model is described in Section 4. The relation from Equation (1) is more complicated for this model and has to be interpolated between those for single-particle and homogeneous sample efficiency. We describe 
the interpolation process using Signal Detection Theory (SDT) [14] in Section 4. For both models, we determine Bias range, Bias standard deviation, and improvement factors from homogeneous calibration. The performance of the $n$-particle model when activities of the particles are not equal is described in Section 5, followed by discussion in Section 6 and conclusions in Section 7. In this work, we are not considering radiation counting statistics and focus exclusively on the dispersion caused by sample inhomogeneity.

\section{Monte Carlo Simulations}

We selected counting geometry for calculations depicted in Figure 1. The sample consists of a 1-L cylindrical container with the radius and height of 5.45 and $10.72 \mathrm{~cm}$, respectively, filled with a soil matrix of $1.55 \mathrm{~g} \cdot \mathrm{cm}^{-3}$ density. A coaxial p-type Ge detector of $48 \%$ relative efficiency was used having crystal radius and height of 3.10 and $5.95 \mathrm{~cm}$, respectively. This configuration actually exists in the laboratory.

The calculations were performed using the MC code Gespecor, version 4.2 (CID Media GmbH, Germany) [15]. This program is especially designed for calculations in gamma spectrometry. It incorporates all materials and dimensions, such as the container, sample, Ge detector, including its housing and dead layer, as well as the lead shield (not shown in Figure 1). The program tracks emitted gamma-rays in all directions. Therefore, the calculations are realistic representations of the laboratory measurement system. Typically, $10^{6}$ events were calculated for each simulation case.

In gamma spectrometry, the effective peak efficiency $p$ at given gamma energy can be expressed as

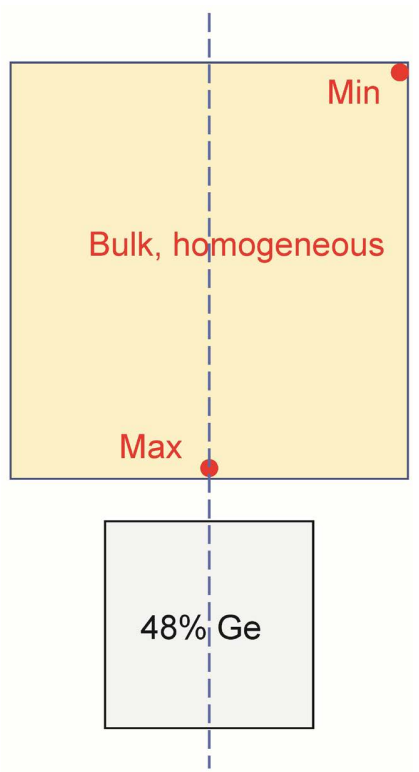

Figure 1. Counting geometry subject of Monte Carlo simulations: 1-L cylindrical sample container and $48 \%$ Ge crystal. The relative dimensions are to scale. The cylindrical symmetry axis is depicted by the broken line. 


$$
p=\varepsilon I F_{c} .
$$

In Equation (2), $\varepsilon$ is a calculated gamma efficiency at that energy independent of radionuclide but including self-absorption in the sample. $I$ is the emitted gamma intensity for a radionuclide such as from Table $1 . F_{c}$ is a calculated coincidence-summing factor dependent on the decay scheme of radionuclide.

In the initial simulations, we calculated the efficiencies for several special cases listed in Table 2 at the three gamma energies of 121.8, 344.3, and $1408.0 \mathrm{keV}$ from ${ }^{152} \mathrm{Eu}$ abbreviated as E1, E2, and E3, respectively. The results are given for hypothetical hot particle locations in Figure 1 resulting in maximum and minimum efficiencies. It is seen that the ratio of effective efficiencies at those locations varies from 35.8 to 250.2 as gamma energy decreases. This can create significant Bias in the determination of hot-particle activity. Also given in Table 2 are efficiencies calculated separately for homogeneous samples, referred to as Bulk efficiencies. It can be observed from Table 2 that the calculated coincidence-summing factors have the lowest values (highest corrections) at the closest distance to the detector and they do not correlate with the gamma energy.

To study the effects associated with hot particles, we calculated the effective peak efficiencies for 2048 particles randomly positioned in the 1-L container, one particle at a time. The random positions of particles were calculated first using the algorithm for cylindrical coordinates [16]. In this algorithm, the height of particle position is proportional to a random number, while the radius of particle position is proportional to the square root of the random number. The azimuth angle is not important in this case because of cylindrical symmetry. Then, the 2048 random particle positions were supplied to the Gespecor program, which calculated effective peak efficiencies at these positions. To verify the randomness of particle positions, we calculated the Grand average efficiencies for all 2048 particles. It is seen from Table 2 that the deviations of Grand average efficiencies from the Bulk efficiencies are between $-1.02 \%$ and $0.67 \%$ for the

Table 2. MC results of special cases for ${ }^{152} \mathrm{Eu}$.

\begin{tabular}{|c|c|c|c|c|c|c|}
\hline $\begin{array}{c}\text { Gamma } \\
\text { efficiency type }\end{array}$ & $\begin{array}{c}\text { Particle location } \\
\text { Radius, Height }(\mathrm{cm})\end{array}$ & Peak & $\begin{array}{c}\text { Coincidence } \\
\text { summing factor }\end{array}$ & $\begin{array}{c}\text { Gamma } \\
\text { efficiency (a) }\end{array}$ & $\begin{array}{l}\text { Effective } \\
\text { efficiency }\end{array}$ & $\begin{array}{c}\text { Effective efficiency ratio } \\
\text { Max/Min }\end{array}$ \\
\hline \multirow{3}{*}{ Maximum } & \multirow{3}{*}{0,0} & E1 & 0.7569 & $2.054 \mathrm{E}-01$ & $4.418 \mathrm{E}-02$ & 250.2 \\
\hline & & E2 & 0.8645 & $9.840 \mathrm{E}-02$ & $2.262 \mathrm{E}-02$ & 104.0 \\
\hline & & E3 & 0.7747 & $3.062 \mathrm{E}-02$ & $4.945 \mathrm{E}-03$ & 35.8 \\
\hline \multirow{3}{*}{ Minimum } & \multirow{3}{*}{$5.45,10.72$} & E1 & 0.9939 & $6.253 \mathrm{E}-04$ & $1.766 \mathrm{E}-04$ & \multirow{3}{*}{$\begin{array}{c}\text { Grand average } \\
\text { deviation from Bulk (\%) }\end{array}$} \\
\hline & & $\mathrm{E} 2$ & 0.9959 & $8.214 \mathrm{E}-04$ & $2.175 \mathrm{E}-04$ & \\
\hline & & E3 & 0.9989 & $6.642 \mathrm{E}-04$ & $1.383 \mathrm{E}-04$ & \\
\hline \multirow{3}{*}{ Bulk } & \multirow{3}{*}{ homogeneous source } & E1 & 0.9169 & $1.553 \mathrm{E}-02$ & $4.045 \mathrm{E}-03$ & 0.52 \\
\hline & & $\mathrm{E} 2$ & 0.9515 & $9.921 \mathrm{E}-03$ & $2.510 \mathrm{E}-03$ & 0.67 \\
\hline & & E3 & 0.9563 & $4.218 \mathrm{E}-03$ & $8.410 \mathrm{E}-04$ & -1.02 \\
\hline
\end{tabular}

a: Corrected for self-absorption in the sample. 
three gamma energies. Therefore, the randomness of particle positions is judged satisfactory, and we will use the Grand average instead of Bulk as the value for homogeneous sample (this differs from the previous work, where we used the Bulk efficiencies [1]).

The wide range of possible effective efficiencies of hot-particles can result in a large Bias of measured sample activity if homogeneous efficiency is inadvertently assumed. Let us abbreviate counting rate in the gamma peak as $R$. The true activity determined using particle effective peak efficiency $p$ is equal to $A=R / p$. The activity determined using homogeneous peak efficiency $p_{h}$ is equal to $A_{h}=$ $R / p_{h}$. Bias is defined as

$$
\operatorname{Bias}(\%)=100\left(A_{h} / A-1\right)=100\left(p / p_{h}-1\right) .
$$

It is seen that the Bias does not depend on the activity, only on the efficiencies. Therefore, this investigation focuses on the efficiencies only. The Bias was calculated using Equation (3) for the 2048 particles and is plotted in Figure 2 for E1 gamma peak. It ranges from about $-100 \%$ to over $900 \%$, or by a factor of about 10. The Bias distribution is non-symmetric as discussed in detail in [1]. This simulated Bias variation is substantially smaller than the peak efficiency ratio at maximum and minimum positions equal to 250.2 (Table 2), however, it is statistically unlikely to have a particle located at any of these positions (compare Figure 1).

\section{One-Particle Model}

One-particle model assumes presence of a single hot particle in the sample. We developed this model as a foundation for easier derivation of a more general

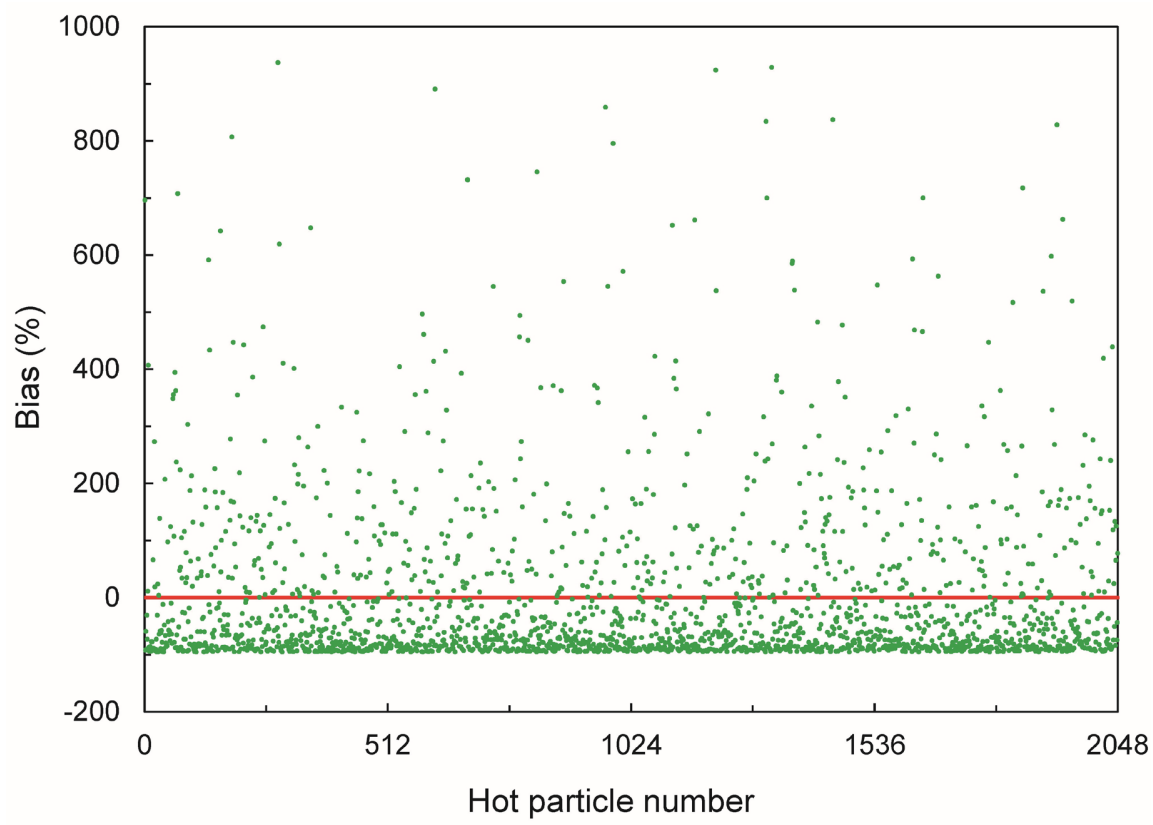

Figure 2. Histogram of Bias of using Grand average efficiency from single particle efficiency for E1 peak of ${ }^{152} \mathrm{Eu}$. 
n-particle model to be described in the next section. Let us consider a particle in the sample matrix located at a distance $r$ from the Ge detector. In a simplified picture, we neglect all possible angles and finite sizes of both sample and the detector. For a gamma radiation to be detected in the peak with effective peak efficiency $p_{r}$, it must be attenuated in the sample and detected with an intrinsic peak efficiency of the detector $p_{d \cdot}$. The coincidence summing is neglected at this stage. For a gamma ray $i$ emitted from the particle at the distance $r$, we thus have

$$
p_{r i}=I_{i} p_{d i} \exp \left(-\mu_{i} r\right),
$$

where $\mu_{i}$ is a total gamma attenuation coefficient [13].

By manipulating Equation (4) for two gamma rays $i$ and $j$ emitted from the same radionuclide at the distance $r$, one obtains

$$
p_{r i}=I_{i} p_{d i}\left(\frac{I_{j} p_{d j}}{I_{i} p_{d i}} \frac{p_{r i}}{p_{r j}}\right)^{\mu_{i} /\left(\mu_{i}-\mu_{j}\right)} .
$$

It is seen from Equation (5) that the effective peak efficiency is a power function of the ratio of two effective peak efficiencies,

$$
p_{r i}=a\left(p_{r i} / p_{r j}\right)^{b},
$$

where $a, b$ are coefficients. Therefore, this ratio carries the information about the location of emission source within the sample, from which true $p_{r i}$ can be computed.

In Figure 3, we plotted the effective peak efficiencies for peaks E1 and E2 as functions of peak ratios, where we always take the ratio of lower to higher energy peak. This results in 3 possible combinations. The MC data for 2048 particles are indicated as shaded areas in Figure 3. For $p_{1}$ vs. $p_{1} / p_{3}$ only, we fitted the data with the power-law function given by Equation (6) (dashed curve).

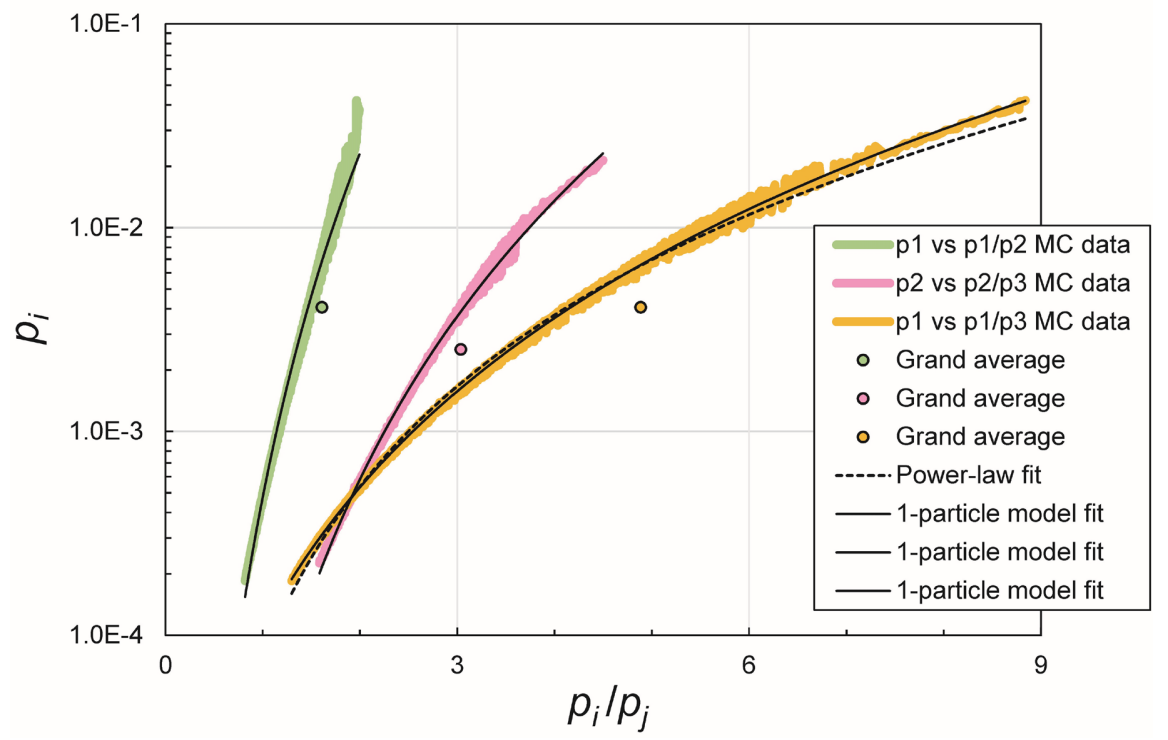

Figure 3. Single-particle calibration curves of effective peak efficiency vs. effective peak efficiency ratio for 3 peaks from ${ }^{152} \mathrm{Eu}$. 
This model can be refined by including coincidence-summing corrections. In general, gamma rays $i$ and $j$ can be coincident with each other as well as many other gamma rays in the decay scheme. The full correction includes all combinations of gamma rays [17]. However, as a first-order approximation, we assume that gamma rays $i$ and $j$ are coincident with each other only. There are two effects to consider. One is called summing-out, in which the intensity of gamma ray $i$ decreases due to coincidence with gamma ray $j$ and vice versa. The second effect is summing-in, where intensity of a third peak (if it exists), with the energy equal to the sum of gamma energies of peaks $i$ and $j$, is increased. Analytical formulas for these two cases were derived [18] [19]. The most common coincidence effect is summing-out, for which the coincidence-summing factor can be written as

$$
F_{c i}=1-c t_{r j}<1,
$$

where $t_{r j}$ is the total efficiency and $c$ is a coefficient. It is seen from Table 2 that the coincidence-summing factors for the peaks from ${ }^{152} \mathrm{Eu}$ are of the summingout category.

Using Equation (7), Equation (4) is refined as

$$
p_{r i}=I_{i} p_{d i} \exp \left(-\mu_{i} r\right)\left(1-c t_{r j}\right) .
$$

By manipulating Equation (8) for two gamma rays $i$ and $j$ emitted from the same radionuclide at the distance $r$, one obtains

$$
p_{r i}=I_{i} p_{d i}\left(1-c t_{r j}\right)\left(\frac{I_{j} p_{d j}}{I_{i} p_{d i}} \frac{p_{r i}}{p_{r j}} \frac{1-d t_{r i}}{1-c t_{r j}}\right)^{\mu_{i} /\left(\mu_{i}-\mu_{j}\right)},
$$

where $d$ is another coefficient. To simplify Equation (9), we abbreviate $y=p_{r i}$ and $x=p_{r i} / p_{r r^{\prime}}$ We also recall from the former investigation [1], that $t$ is a power function of $p$, whereas $p$ is a power function of $x$ according to Equation (6). Therefore, $t$ is also a power function of $x$, different for peaks $i$ and $j$. Equation (9) can be rewritten as

$$
y=a\left(1-b x^{c}\right)\left(x \frac{1-d x^{e}}{1-b x^{c}}\right)^{f}
$$

where $a$ through $f$ is another set of coefficients. Equation (10) is ill-defined for fitting purposes because coefficients $b$ and $c$ occur more than once. To simplify this, we note that the ratio of summing-out factors in the fraction tends to cancel out, as can be seen in the examples from Table 2. Therefore, we neglect these factors and arrive at the final simplified equation:

$$
y=a\left(1-b x^{c}\right) x^{d}
$$

with fit coefficients $a, b, c$, and $d$.

We fitted Equation (11) to $p_{1}$ vs. $p_{1} / p_{3}$ data in Figure 3 (solid curve) resulting in a slightly better fit than that using Equation (6) (dashed curve). Other cases in Figure 3 were fitted with Equation (11) as well; they appear to be slightly worse 
than the $p_{1}$ vs. $p_{1} / p_{3}$ fit. These fits are the calibration curves for the 1-particle model.

Then, we calculated the Bias values from the true single-particle efficiencies when using 1-particle model for E1 peak calibrated with E1/E3, for 2048 particles. Equation (3) was modified by replacing $p_{h}$ with $p_{1 \text {-particle }}$ for the 1-particle model.

$$
\operatorname{Bias}(\%)=100\left(p / p_{1-\text { particle }}-1\right) .
$$

It is seen from Figure 4 that the Bias is significantly reduced to about $\pm 20 \%$. We calculated the Bias Range (lowest value of Bias subtracted from the highest value) and Bias Std Dev with the 1-particle model for each of the 3 cases studied using the calibration curves from Figure 3. Also, the Bias Range and Bias Std Dev were calculated for homogeneous calibration (for the data depicted in Figure 2). The Improvement factor is defined as a Bias measure for homogeneous calibration divided by the corresponding measure for the 1-particle model. Improvement factors indicate how many times 1-particle model performs better than the homogeneous calibration, when measuring randomly distributed single hot particle in the sample. It is seen from Figure 5 that the Improvement factors are significant. The Improvement factors for both Bias Range and Bias Std Dev are the highest when the gamma energy difference between the peaks in the largest (E1, E3), whereas they are the lowest for $(\mathrm{E} 1, \mathrm{E} 2)$.

\section{4. $n$-Particle Model}

The $n$-particle model assumes that there are 1 or more hot particles in the sample, all having the same activity. It can be realized by simple grouping of the

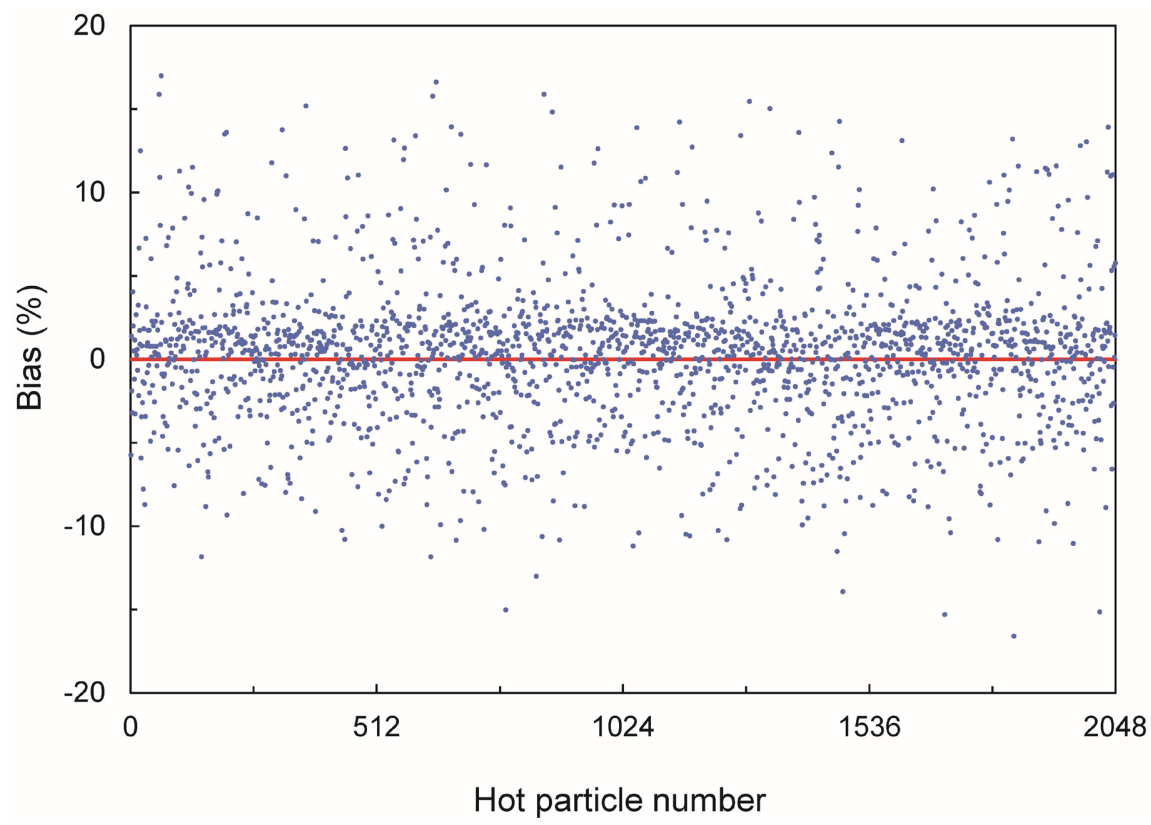

Figure 4. Histogram of Bias of using 1-particle model efficiency from single particle efficiency for E1 peak of ${ }^{152} \mathrm{Eu}$. 


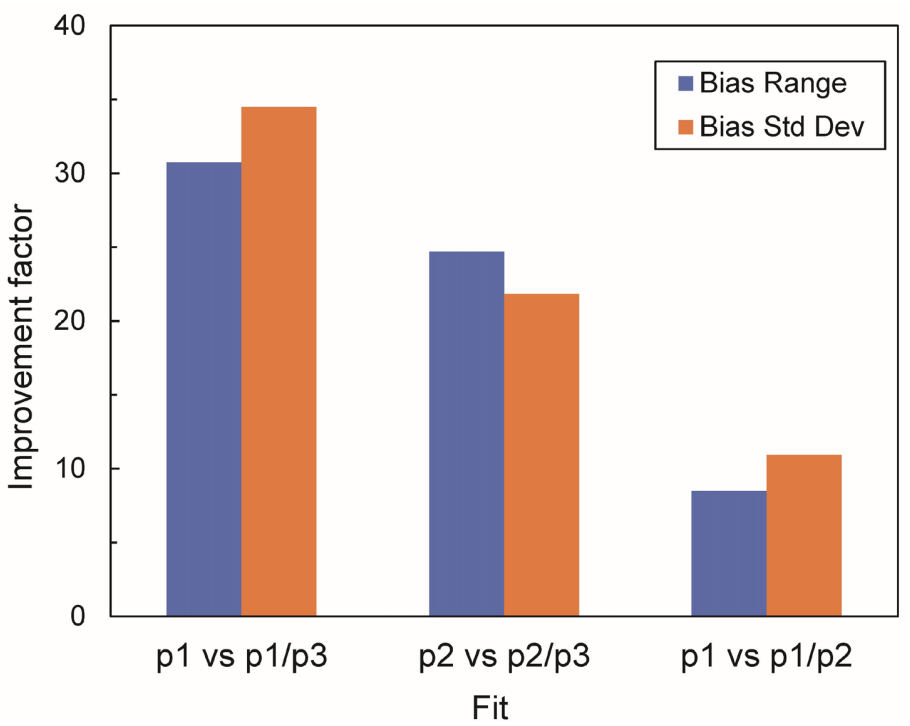

Figure 5. Improvement factors of Bias range and Bias Std Dev by 1-particle model from homogeneous model for various combinations of peaks.

2048 MC particles. For instance, one can group 2 particles (1024 cases), 4 particles (512 cases), etc., until finally arriving at 1 case of 2048 particles. The average efficiencies and their ratio for any group of $n$ particles are given by

$$
\begin{gathered}
\left\langle p_{i}\right\rangle_{n}=\frac{1}{n} \sum_{k=1}^{n} p_{k i}, \\
\left\langle p_{i} / p_{j}\right\rangle_{n}=\left\langle p_{i}\right\rangle_{n} /\left\langle p_{j}\right\rangle_{n} .
\end{gathered}
$$

As an example, the $\left\langle p_{1}\right\rangle_{n}$ vs. $\left\langle p_{1} / p_{3}\right\rangle_{n}$ groups are plotted in Figure 6. They form fanning-out bands. The band at $n=1$ is the topmost, identical to the data in Figure 3. As $n$ increases, the bands are becoming narrower and positioned lower in Figure 6, until they reach the Grand average point at $\left\langle p_{1}\right\rangle_{2048}$ for $\left\langle p_{1} / p_{3}\right\rangle_{2048}$, which we take as the point of the homogeneous calibration, $p_{h}$.

The Bias of the homogeneous calibration for the grouped particles is generalized from Equation (3), with $p$ substituted by $\langle p\rangle_{n}$ as follows:

$$
\operatorname{Bias}(\%)=100\left(\langle p\rangle_{n} / p_{h}-1\right) .
$$

The Bias results of homogeneous calibration for E1 peak are given in the upper part of Table 3, for groups up to 512 particles. The Bias Std Dev and Bias Range for 1 hot particle are for the data from Figure 2. It is seen that as the number of particles in a group increases, the Bias Std Dev and Bias Range decrease. The characteristic result of homogeneous calibration is that the Average Bias is zero for all particle groups. This reflects the fact that if sufficient number of repetitions are performed, the Average Bias tends to zero, and it is the basis for sample splitting and mixing methods described in Section 1.

The first step of the n-particle model is to encompass the points in Figure 6. The upper bound is given by the 1-particle model fit which is the black curve in 
Table 3. ${ }^{152} \mathrm{Eu}$ E1 peak Bias using the homogeneous calibration and Bias using $n$-particle model for E1/E3.

\begin{tabular}{ccccccccccc}
\hline \multirow{2}{*}{$\begin{array}{c}\text { Bias } \\
\text { measure }\end{array}$} & 1 & 2 & 4 & 8 & 16 & 32 & 64 & 128 & 256 & 512 \\
\cline { 2 - 11 } & \multicolumn{8}{c}{ Homogen } & \multicolumn{10}{c}{ Number of hot particles $n$} \\
\hline Average & 0.0 & 0.0 & 0.0 & 0.0 & 0.0 & 0.0 & 0.0 & 0.0 & 0.0 & 0.0 \\
Std Dev & 146.9 & 102.1 & 75.1 & 53.5 & 38.1 & 26.7 & 19.1 & 14.1 & 10.2 & 2.4 \\
Range & 1032.5 & 681.5 & 442.0 & 294.6 & 175.6 & 113.7 & 79.0 & 54.4 & 26.6 & 5.3 \\
\hline & & & $n-$ particle model Bias (\%) & & & & \\
\hline Average & 14.9 & 3.3 & -3.7 & -4.9 & -3.4 & -0.5 & 0.8 & 0.4 & 0.2 & 0.0 \\
Std Dev & 27.8 & 25.2 & 23.6 & 21.5 & 17.9 & 10.8 & 5.9 & 4.2 & 2.6 & 0.8 \\
Range & 120.3 & 137.6 & 131.3 & 108.9 & 86.2 & 54.4 & 21.7 & 15.3 & 8.5 & 1.8 \\
\hline
\end{tabular}

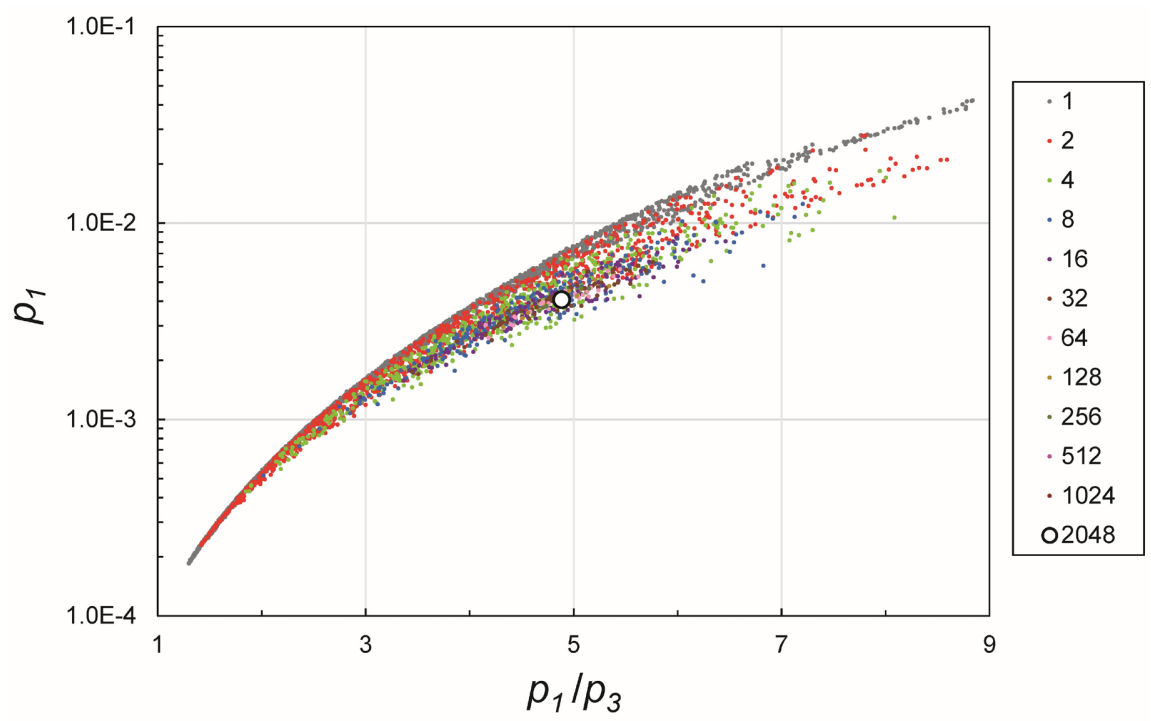

Figure 6. Distribution of $\left\langle p_{1}\right\rangle_{n}$ peak efficiency as a function of $\left\langle p_{1} / p_{3}\right\rangle_{n}$ peak efficiency ratio for grouped hot particles $n$ indicated in the legend.

Figure 7, replotted from Figure 3. To construct the lower bound, we took the points of minimum and maximum values of $\left\langle p_{1} / p_{3}\right\rangle_{n}$ for each $n$-particle set (they are unrelated to the Min and Max points in Figure 1). These are the blue points in Figure 7. Equation (11) was then fitted to the Min-Max points, constrained by the passage through the Grand average point, which resulted in an orange curve in Figure 7. It is seen that most of the points from Figure 6 are encompassed by the two fits: 1-particle model curve and Min-Max curve.

In order to use the model for any number of hot particles possibly present in the sample, one needs to interpolate between the two curves in Figure 7. The reason for that is that when $p_{1} / p_{3}$ is close to the Grand average, we likely have a more homogeneous sample containing more hot particles, so the interpolation weights $p_{1}$ towards Min-Max curve. On the contrary, when $p_{1} / p_{3}$ is away from 


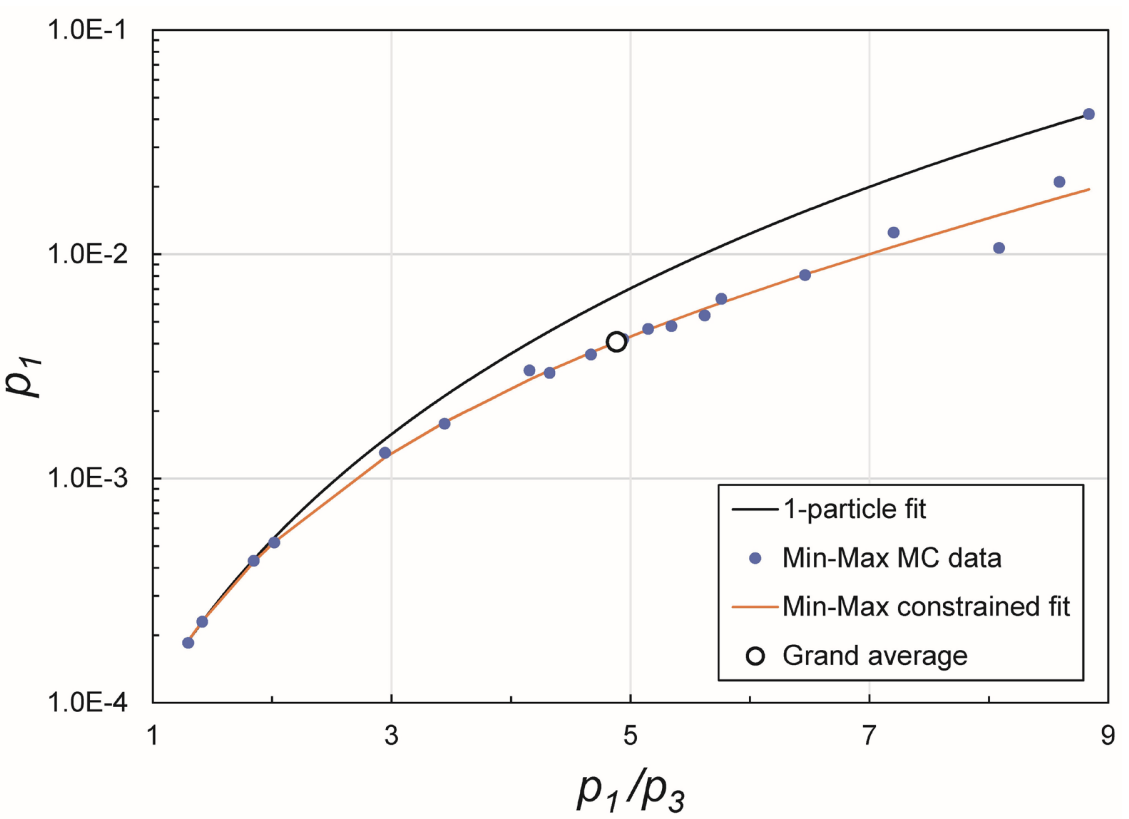

Figure 7. Various fits to $\left\langle p_{1}\right\rangle_{n}$ vs. $\left\langle p_{1} / p_{3}\right\rangle_{n}$ data for ${ }^{152} \mathrm{Eu}$ : 1-particle model (black curve), Min-Max (orange curve).

the Grand average, we likely have an inhomogeneous sample containing a smaller number of hot particles (or only 1 ), so the interpolation weights $p_{1}$ towards 1-particle curve.

For the interpolation, we applied the SDT technique developed elsewhere [1] [14]. The equations are identical to the ones presented in [1] and will not be repeated here. As a result of the interpolation, the most optimal $p_{1}$ can be obtained abbreviated as $p_{\text {interp }}$. The performance of the interpolation can be adjusted by the mean and sigma parameters of the signal in SDT: $\mu_{\text {signab }} \sigma_{\text {signal }}$. The Bias for the $n$-particle model is given by

$$
\operatorname{Bias}(\%)=100\left(\langle p\rangle_{n} / p_{\text {interp }}-1\right) \text {. }
$$

As an example, the Bias Average, Std Dev, and Range for a particular $n$-particle model fit to (E1, E3) case are given in the lower section of Table 3. There is only one fit for all groups of hot particles. There is no absolute minimum here and the model parameters, $\mu_{\text {signas }} \sigma_{\text {signas }}$ are adjusted manually to achieve desired data objectives. One noticeable property of the $n$-particle model is that the Average Bias is not zero for most particle groups. This causes systematic Bias in the results. A chosen data objective was to have this systematic Bias within the range of $\pm 5 \%$ for as many hot particle groups as possible. As can be seen from Table 3, this objective is satisfied for all particle groups, except for 1 particle, where the Average Bias is $14.9 \%$. We attribute this to the fact that the Grand average point lays quite away from the 1-particle model calibration, which can be seen in Figure 3, Figure 6, and Figure 7.

The significant advantages of the $n$-particle model are in the reductions of Bias Range and Bias Std Dev compared with the homogeneous calibration, as 
demonstrated in Table 3 . We fitted the $n$-particle model to the 3 combination of gamma peaks studied: (E1, E3), (E2, E3), and (E1, E2). Then we calculated the Improvement factors from the homogeneous calibrations, as described in Section 3. The Improvement factors for Bias Range are depicted in Figure 8. They decrease from about a factor of 8 to about a factor of 2 as the number of hot particles in a group increases. The slight increase at 64 particles is attributed to a sharper transition in the interpolation from 1-particle model to Min-Max, as described above Equation (16). The improvement factors for Bias Range vary between 5 and 2 (Figure 9). One feature seen in Figure 8 and Figure 9 is that

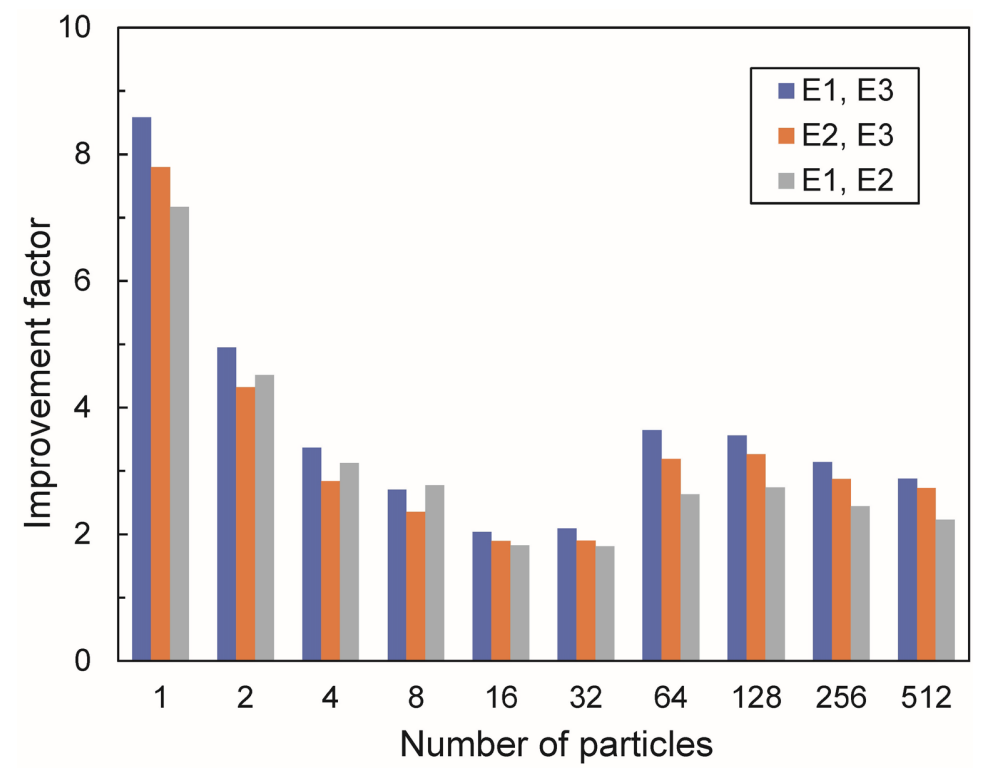

Figure 8. Bias range improvement factors of $n$-particle model from homogeneous model for various combinations of peaks from ${ }^{152} \mathrm{Eu}$ indicated in the legend.

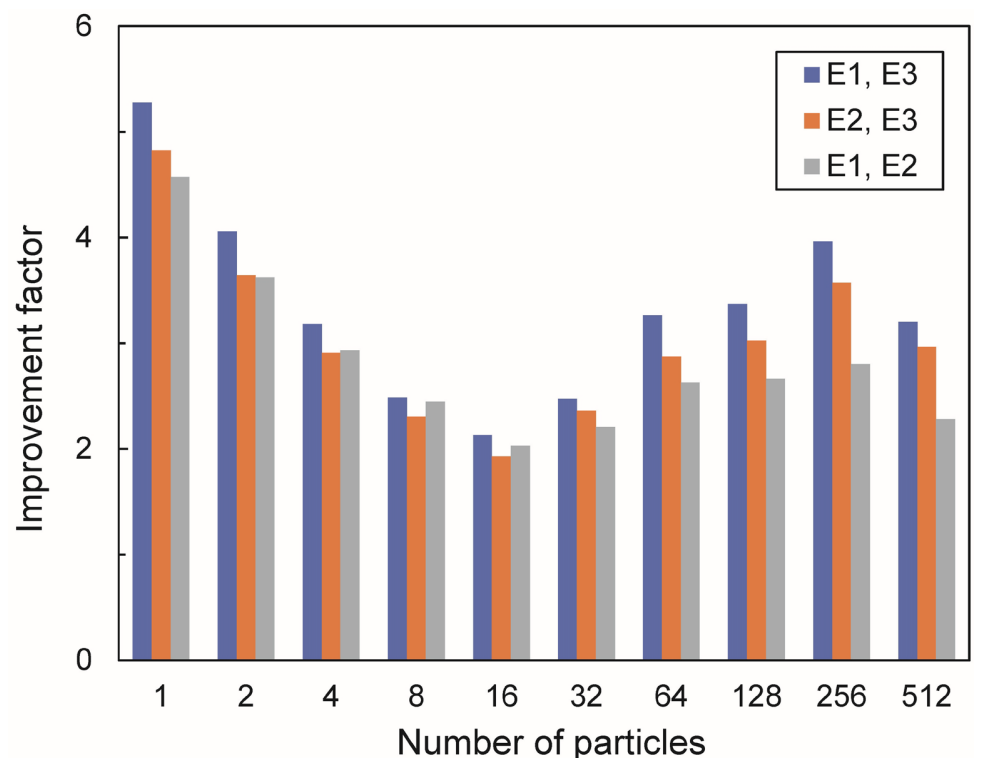

Figure 9. Bias Std Dev improvement factors of $n$-particle model from homogeneous model for various combinations of peaks from ${ }^{152} \mathrm{Eu}$ indicated in the legend. 
there are only minor differences between the 3 cases of peaks studied. This is in contrast to the observation in Figure 5 for 1-particle model, where Improvement factors depended significantly on the energies of gamma peaks.

\section{Non-Equal Particles}

The $n$-particle model assumes that all particles have the same activities. It was observed that hot particles from nuclear accidents exhibit a distribution of sizes often consistent with lognormal [3]. Then, an assumption of constant specific activity implies lognormal distribution of activities as well. This situation results in even more inhomogeneity than for equal particles, because a few very hot particles dominate the activity of the sample.

Non-equal particles can be easily simulated within the present data set by positioning several equal particles in the same location. Since the derived $n$-particle model interpolates the efficiencies between 1 particle and the homogeneous sample, it should be independent of a specific assumption about the number or location of the particles. Therefore, it should accommodate non-equal particles as well.

To test this hypothesis, we created three cases of non-equal particles. In the first case, we have 2 particles, one is assumed twice as radioactive as the other. Therefore, the average peak efficiency from Equation (13) is $\langle p\rangle_{2}=\left(2 p_{1}+p_{2}\right) / 3$. We have used 2 equal particles from the MC set of 2048 and created a group of 2 non-equal particles. We have 1024 such groups to perform statistics on. For the second case, we repeated $1^{\text {st }}$ particle 4 times, $2^{\text {nd }}$ particle 2 times, and took the $3^{\text {rd }}$ and $4^{\text {th }}$ particles as is. The average peak efficiency is $\langle p\rangle_{4}=\left(4 p_{1}+2 p_{2}+p_{3}+p_{4}\right) / 8$. We thus have 512 such groups. In the third case $\langle p\rangle_{8}=\sum_{k=1}^{8} k p_{k} / 36$, with 256 groups.

Following this, the Bias of the homogeneous calibration was calculated from Equation (15) for each of the non-equal particle cases. By applying the $n$-particle model as in Section 3, the Bias was calculated from Equation (16). Then the Improvement factors of the $n$-particle model from the homogeneous model were calculated and are depicted in Figure 10. For the Bias Range, the Improvement factor decreased from almost 5 to over 3, whereas for the Bias Std Dev from about 3.5 to 3 . These values are close to those for 2,4 , and 8 equal particles. Therefore, the $n$-particle model works for non-equal particles as well.

\section{Discussion}

Voluminous environmental or food samples for gamma spectrometry measurements in the laboratory can be quite inhomogeneous. The inhomogeneity is typically caused by the presence of hot particles, where radioactivity is contained within micrometer-size particles originating from nuclear reactor accidents and fires, nuclear explosions, or dirty-bomb explosions. Hot particles are often refractory and difficult to homogenize. Mechanical mixing only repositions the hot particles in the sample without homogenizing them. However, gamma spectrometers are typically calibrated using either homogeneous physical standards 


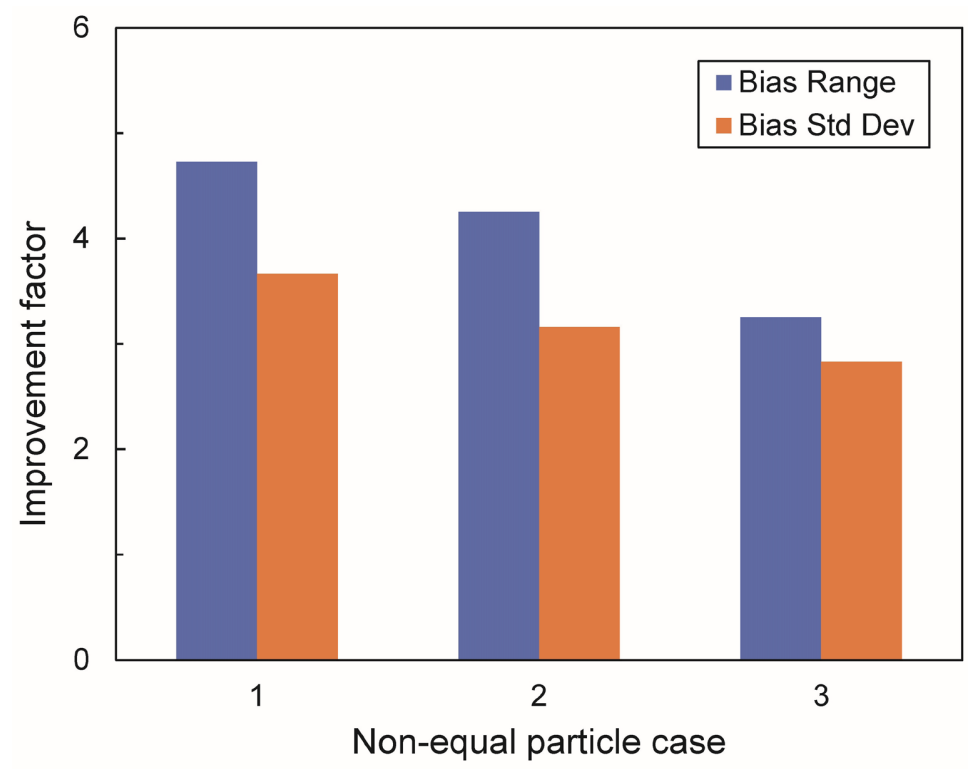

Figure 10. Improvement factors of Bias range and Bias Std Dev provided by the $n$-particle model from the homogeneous model for three cases of non-equal hot particles studied.

or by $\mathrm{MC}$ methods assuming uniform distribution of radioactivity, which results in a homogeneous or bulk effective efficiency $p_{h}$. Applying $p_{h}$ to inhomogeneous samples can lead to significant Bias of measured activity. One approach to address this is by remixing and remeasuring the sample at least several tens of times. The statistics of large number of mixings will result in a relatively accurate mean activity, however, the dispersion evaluated by means of a standard deviation can be large and increase as inhomogeneity increases. This implies that a single measurement of inhomogeneous sample can likely lead to inaccurate activity determination, which can differ from a true activity by several times.

In this investigation, we succeeded in developing a method to reduce this dispersion. If a radionuclide has several prominent gamma peaks $i$ which result in measured counting rates $R_{p}$ the radionuclide activities determined from those peaks are equal to $A_{h i}=R_{i} / p_{h i}$ where $p_{h i}$ is effective homogeneous efficiency for peak $i$. If the sample is homogeneous, all $A_{h i}$ should be equal to within experimental uncertainties. Significant differences between $A_{h i}$ indicate inhomogeneity. Since the counting rates are proportional to the effective efficiencies, it follows that the ratio of two peak counting rates (or peak areas) is proportional to the ratio of effective true efficiencies $p_{i} / p_{j}$. Therefore, the latter is a measure of inhomogeneity. This is key to the principle of the peak-ratio method.

In order to quantify the peak-ratio method, we derived the equation showing that $p_{i}$ is a power function of $p_{i} / p_{p}$ including coincidence-summing corrections. We verified the new method by MC simulation. A sample of soil in 1-L cylindrical container was assumed measured on a $48 \%$ efficient p-type Ge detector. We simulated random locations of 2048 hot particles in the sample, one particle at a time, and calculated their effective efficiencies and efficiency ratios for three 
gamma peaks of ${ }^{152} \mathrm{Eu}: \mathrm{E} 1=121.8 \mathrm{keV}, \mathrm{E} 2=344.3 \mathrm{keV}$, and $\mathrm{E} 3=1408.0 \mathrm{keV}$. The simulated functions $p_{1}$ vs. $p_{1} / p_{3}, p_{2}$ vs. $p_{2} / p_{3}$, and $p_{1}$ vs. $p_{1} / p_{2}$ were fitted with the derived equation resulting in the 1-particle calibration model. This model was applied to calculate Bias of the fitted curve from true values of $p_{i}$ for each particle, in terms of Bias Range and Bias Std Dev. Then the Improvement factors were calculated for the Bias measures with respect to the Bias measures assuming homogeneous calibrations. Spectacular Improvement factors were determined for the peak-ratio method in the presence of 1 particle in the samples. The improvement factors decreased from about 30 to 20 to 10 when moving from E1/E3 to E2/E3 to E1/E2 calibrations. Therefore, the larger the difference between the energies of the two peaks, the better the Improvement factor is, at least for a single particle present in the sample.

However, this method does not aim at detecting exactly 1 hot particle in the sample.

Therefore, identical individual particles were combined into $n$-particle groups in steps of 2 , which resulted in the $n$-particle model. For each group, the average effective peak efficiencies $\left\langle p_{i}\right\rangle_{n}$ and their ratios were calculated. It was observed that $\left\langle p_{i}\right\rangle_{n}$ vs. $\left\langle p_{i} / p_{j}\right\rangle_{n}$ curves were lower than that for the 1-particle. In order to overlap all grouped MC data, the Minimum and Maximum values of $p_{i} / p_{j}$ were extracted for each $n$, which resulted in the Min-Max calibration curve constrained by passing through the Grand average point. The $n$-particle calibration curve was interpolated between 1-particle and Min-Max curves, using SDT, leading to the most optimal effective efficiency $p_{\text {interp }}$, for every $n$. Then the Bias Range and Bias Std Dev of $p_{\text {interp }}$ from that of true efficiency $\left\langle p_{i}\right\rangle_{n}$ was calculated as well as the Improvement factors from the homogeneous efficiency. The Improvement factors varied from 8 to 2 for the Bias Range and from 5 to 2 for the Bias Std Dev when increasing the number of hot particles in the group. In comparison with the 1-particle model, the Improvement factors are lower, and their dependence on gamma energy is much less.

We also tested the model for non-equal particles. We simulated 3 groups of non-equal hot particles and found that the $n$-particle model provided expected Improvement factors from the homogeneous calibration. Therefore, this model is independent of the number, location, and size distribution of hot particles.

The application of our calibrated $n$-particle model to determine radionuclide activity can be as follows. The ratio of two peak areas or counting rates of the radionuclide is determined from the measured gamma spectrum. Using this ratio, the effective peak efficiency $p_{i}$ is calculated from the $p_{i}$ vs. $p_{i} / p_{j}$ interpolated calibration curve. Then, the radionuclide activity is calculated as the $A_{i}=R_{i} / p_{i}$. Our calculated Improvement factors suggest that, in a single measurement of a random sample, the $n$-particle model is expected to deliver activity result several times closer to true activity than that calculated using bulk homogeneous model. In this way, $n$-particle model satisfies the stated goal of reducing dispersion in gamma spectrometry of inhomogeneous samples. One parameter the $n$-particle model cannot provide is the standard deviation of inhomogeneity itself. Actual- 
ly, it would, if the number and distribution of hot-particles was known, which is not available from a single measurement.

The advantage of the $n$-particle model is that can be applied to a mixture of radionuclides, as long as each radionuclide has at least two gamma peaks easily measurable in the gamma spectrum. Table 1 lists several radionuclides to which $n$-particle model can be applied. There are some limitations attributed to gamma energies and intensities. One such case is ${ }^{137} \mathrm{Cs}$, which has a $32.1-\mathrm{keV}$ x-ray and a $661.7-\mathrm{keV}$ gamma ray emitted by its progeny, ${ }^{137} \mathrm{Ba}$. The low-energy $\mathrm{x}$-ray is easily attenuated in the sample, so the sample size has to be smaller than $1 \mathrm{~L}$ (in our configuration on Figure 1), and Ge detector sensitive to low-energy photons to observe required effects. Similarly, the usage of $32-\mathrm{keV}$ peak was found useful only to matrix depth of $2 \mathrm{~cm}$ for in-situ gamma spectrometry [9]. Also, ${ }^{60} \mathrm{Co}$ (not listed in Table 1) is not an optimal case for peak-ratio method because the attenuation of the two high-energy gamma rays emitted at $1173.2 \mathrm{keV}$ and 1332.5 $\mathrm{keV}$ in the sample is similar. This might not provide sufficient variability in the ratio of their counting rates as a function of inhomogeneity.

In the previous investigation [1], we developed a similar model of handling inhomogeneity in gamma spectrometry of voluminous samples based on the peak-to-total ratio, $p / t$. Unlike the peak-ratio model developed here, the $p / t$ model could only be used for single radionuclides, such as ${ }^{137} \mathrm{Cs}$ or ${ }^{60} \mathrm{Co}$. It also delivered lower Improvement factors than the peak-ratio model. The reason for that is a much wider distribution of 1-particle $p / t$ values than the distribution of 1-particle $p_{i} / p_{j}$ values. The narrow distribution of the latter has a disadvantage, however, in causing the Grand average point laying away from it. This complicated the interpolation process for $n$-particle model and caused the Average Bias to deviate from zero. Nevertheless, it was possible to adjust Average Bias to within $\pm 5 \%$ for all cases, except for 1 particle (the $p / t$ model had Average Biases within $\pm 5 \%$ for all cases including that of 1 particle). The $5 \%$ systematic Bias appears acceptable when comparing Bias of several hundred \% due to inhomogeneity. Since in real samples chances of having exactly 1 hot particle are small, the $n$-particle model can have a useful application. If it was known that there was exactly 1 particle in the sample, then the 1-particle model was shown to perform exceptionally well.

Both the peak-ratio model presented here and the peak-to-total model [1] were applied to specific counting geometry and sample matrix composition and density. For other geometries and matrices, the models require additional fits, which can result in significant computational effort. However, the trends, especially for a fixed geometry and varied sample matrix and density, are expected to be similar, so only a reasonably limited number of calibration cases would need to be calculated, and the ones in between-interpolated.

\section{Conclusion}

A new calibration method was developed to address inhomogeneity in volumin- 
ous samples measured by gamma spectrometry, caused by the presence of hot particles. The method is based on the counting-rate ratio of two gamma peaks from a measured radionuclide. With appropriate calibration using Monte Carlo simulation, the peak-ratio method can determine radionuclide activity several times closer to the true value than the traditional calibration assuming homogeneous sample, by performing only a single measurement. The peak-ratio method has been shown to be independent of the number, location, and size distribution of hot particles. This method can be applied to a mixture of radionuclides in the sample and complements previously developed peak-to-total method to correct for inhomogeneity in gamma spectrometry. The models proposed here could be verified by experiments in which one or more artificially created hot particles of known activities could be randomly mixed in the matrix studied.

\section{Acknowledgements}

Partial support of this work by the U.S. FDA FERN LFFM 1U19FD00708901 is acknowledged. Thanks are due to E. Blain for careful reading of the manuscript.

\section{Conflicts of Interest}

The authors declare no conflicts of interest regarding the publication of this paper.

\section{References}

[1] Chu, L.T., Burn, A.G., Bradt, C.J. and Semkow, T.M. (2021) Monte Carlo Simulation Study of Hot-Particle Detection in Voluminous Samples by Gamma Spectrometry. Journal of Applied Mathematics and Physics, 9, 1522-1540. https://doi.org/10.4236/jamp.2021.97104

[2] Bunzl, K. (1997) Probability for Detecting Hot Particles in Environmental Samples by Sample Splitting. Analyst, 122, 653-656. https://doi.org/10.1039/A700252A

[3] Shevchenko, S.V. (2004) On the Uncertainty in Activity Measurements for Samples Containing "Hot Particles". Applied Radiation and Isotopes, 61, 1303-1306. https://doi.org/10.1016/j.apradiso.2004.02.022

[4] Nageldinger, G., Flowers, A., Henry, B. and Postak, J. (1998) Hot Particle Detection Using Uncertainties in Activity Measurements of Soil. Health Physics, 74, 472-477. https://doi.org/10.1097/00004032-199804000-00009

[5] Bunzl, K. (1998) Detection of the Radioactive Hot Particles in Environmental Samples by Repeated Mixing. Applied Radiation and Isotopes, 49, 1625-1631. https://doi.org/10.1016/S0969-8043(98)00002-5

[6] Kashparov, V.A., Yoshchenko, V.I., Levtchuk, S.E., Tschiersch, J. and Wagenpfeil, F. (2000) Application of the Method of Repeated Mixing to Nonuniformity Contaminated Bulky Samples. Journal of Radioanalytical and Nuclear Chemistry, 246, 165-172. https://doi.org/10.1023/A:1006738727056

[7] Rybacek, K., Jacob, P. and Meckbach, R. (1992) In Situ Determination of Deposited Radionuclide Activities: Improved Method Using Derived Depth Distributions from the Measured Photon Spectra. Health Physics, 62, 519-528. https://doi.org/10.1097/00004032-199206000-00004

[8] Thummerer, S. and Jacob, P. (1998) Determination of Depth Distributions of Nat- 
ural Radionuclides with in Situ Gamma-Ray Spectrometry. Nuclear Instruments and Methods in Physics Research Section A, 416, 161-178.

https://doi.org/10.1016/S0168-9002(98)00636-6

[9] Shippen, A. and Joyce, M.J. (2010) Profiling the Depth of Caesium-137 Contamination in Concrete via a Relative Linear Attenuation Model. Applied Radiation and Isotopes, 68, 631-634. https://doi.org/10.1016/j.apradiso.2009.09.046

[10] Trnková, L., Trojek, T. and Thinová, L. (2010) Gamma Spectrometric Measurements of Depth-Related Radionuclide Distribution in Walls. Applied Radiation and Isotopes, 68, 832-835. https://doi.org/10.1016/j.apradiso.2009.09.061

[11] Feng, T.C., Jia, M.Y. and Feng, Y.J. (2012) Method-Sensitivity of in Situ $\gamma$ Spectrometry to Determine the Depth-Distribution of Anthropogenic Radionuclides in Soil. Nuclear Instruments and Methods in Physics Research Section A, 661, 26-30. https://doi.org/10.1016/j.nima.2011.09.014

[12] Haddad, K., Al-Masri, M.S. and Doubal, A.W. (2014) Determination of ${ }^{226} \mathrm{Ra}$ Contamination Depth in Soil Using the Multiple Photopeaks Method. Journal of Environmental Radioactivity, 128, 33-37. https://doi.org/10.1016/j.jenvrad.2013.10.025

[13] Strom, E. and Israel, H.I. (1970) Photon Cross Sections from $1 \mathrm{keV}$ to $100 \mathrm{MeV}$ for Elements $\mathrm{Z}=1$ to $\mathrm{Z}=100$. Atomic Data and Nuclear Data Tables, 7, 565-681. https://doi.org/10.1016/S0092-640X(70)80017-1

[14] Wickens, T.D. (2002) Elementary Signal Detection Theory. Oxford University Press, New York. https://doi.org/10.1093/acprof:oso/9780195092509.001.0001

[15] Sima, O., Arnold, D. and Dovlete, C. (2001) GERPECOR: A Versatile Tool in Gamma-Ray Spectrometry. Journal of Radioanalytical and Nuclear Chemistry, 248, 359-364. https://doi.org/10.1023/A:1010619806898

[16] Sima, O. (2012) Efficiency Calculation of Gamma Detectors by Monte Carlo Methods. In: Encyclopedia of Analytical Chemistry, John Wiley \& Sons, Hoboken, 37 p. https://doi.org/10.1002/9780470027318.a9142

[17] Semkow, T.M., Mehmood, G., Parekh, P.P. and Virgil, M. (1990) Coincidence Summing in Gamma-Ray Spectroscopy. Nuclear Instruments and Methods in Physics Research Section A, 290, 437-444. https://doi.org/10.1016/0168-9002(90)90561-J

[18] McCallum, G.J. and Coote, G.E. (1975) Influence of Source-Detector Distance on Relative Intensity and Angular Correlation Measurements with Ge(Li) Spectrometers. Nuclear Instruments and Methods, 130, 189-197. https://doi.org/10.1016/0029-554X(75)90173-1

[19] Debertin, K. and Schötzig, U. (1979) Coincidence Summing Corrections in Ge(Li)Spectrometry at Low Source-to-Detector Distances. Nuclear Instruments and $\mathrm{Me}$ thods, 158, 471-477. https://doi.org/10.1016/S0029-554X(79)94845-6

[20] (n.d.) Chart of Nuclides, National Nuclear Data Center, Brookhaven National Laboratory, Upton, NY. https://www.nndc.bnl.gov/nudat2/ 